\title{
ilmedia
}

Ilchmann, Achim ; Ryan, E. P.:

On gain adaptation in adaptive control

Zuerst erschienen in:

IEEE Trans. on Autom. Control 48 (2003) S. 895-899

DOI: 10.1109/TAC.2003.811276 


\section{On Gain Adaptation in Adaptive Control}

\author{
A. Ilchmann and E. P. Ryan
}

\begin{abstract}
The adaptive high-gain output feedback strategy $u(t)=$ $-k(t) y(t),(d / d t) k(t)=\|y(t)\|^{2}$ is well established in the context of linear, minimum-phase, $m$-input $m$-output systems $(A, B, C)$ with the property that $\operatorname{spec}(C B) \subset \mathbb{C}_{+}$; the strategy applied to any such linear system achieves the performance objectives of: 1) global attractivity of the zero state and 2) convergence of the adapting gain to a finite limit. Here, these results are generalized in three aspects. First, the class of systems is enlarged to a class $\mathcal{N}_{h}(\mu)$, encompassing nonlinear systems modeled by functional differential equations, where the parameter $h>0$ quantifies system memory and the continuous function $\mu:[0, \infty) \rightarrow$ $[0, \infty)$, with $\mu(0)=0$, relates to the allowable system nonlinearities. Second, the linear control law is replaced by $u(t)=-k(t)[y(t)+$ $\mu(\|y(t)\|) /\|y(t)\|] y(t)$, wherein the additional nonlinear term counteracts the system nonlinearities. Third, the quadratic adaptation law is replaced by the law $(d / d t) k(t)=\psi(\|y(t)\|)$, where the continuous function $\psi$ satisfies certain growth conditions determined by $\mu$ (in particular cases, e.g., linear systems, a bounded function $\psi$ is admissible). Performance objectives 1) and 2) above are shown to persist in the generalized framework.
\end{abstract}

Index Terms-Adaptive control, functional differential equations, minimum-phase systems.

\section{INTRODUCTION}

Consider the class $\mathcal{L}$ of finite-dimensional, real, linear, minimumphase, $m$-input $\left(u(t) \in \mathbb{R}^{m}\right), m$-output $\left(y(t) \in \mathbb{R}^{m}\right)$ systems of the form

$$
\left.\begin{array}{l}
\dot{x}(t)=A x(t)+B u(t)+p(t), \quad x(0)=x^{0} \\
y(t)=C x(t) \\
\operatorname{det}\left[\begin{array}{cc}
s I_{n}-A & B \\
C & 0
\end{array}\right] \neq 0 \forall s \in \overline{\mathbb{C}}_{+} \\
\operatorname{spec}(C B) \subset \mathbb{C}_{+}
\end{array}\right\}
$$

with $n, m \in \mathbb{N}, n \geq m, x^{0} \in \mathbb{R}^{n}$, and where $p \in L^{2}\left(\mathbb{R}_{\geq 0} ; \mathbb{R}^{n}\right)$ is a perturbation, $(A, B, C)$ is a triple of real matrices of conforming formats, $\mathbb{R}_{\geq 0}:=[0, \infty)$ and $\mathbb{C}_{+}:=\{\lambda \in \mathbb{C} \mid \operatorname{Re}(\lambda)>0\}$ denotes the open right-half complex plane, with closure $\overline{\mathbb{C}}_{+}$. The condition on the determinant in (1) characterizes the minimum-phase assumption and the spectrum condition $\operatorname{spec}(C B) \subset \mathbb{C}_{+}$is a multiple-input-multipleoutput counterpart of the "positive high-frequency gain" assumption for single-input-single-output systems.

As is well known (see, for example, the seminal work in [1], [3], and [4]), the adaptive output feedback control

$$
u(t)=-k(t) y(t) \quad \dot{k}(t)=\|y(t)\|^{2}, \quad k(0)=k^{0} \in \mathbb{R}
$$

is an $\mathcal{L}$-universal stabilizer in the sense that the control, applied to any member of the class $\mathcal{L}$, ensures that: 1 ) the zero state is globally attractive and 2) the adapting gain converges to a finite limit. Whilst simple, the quadratic nature of the gain adaptation law in (2) can result in intervals of rapid increase in gain which potentially generate asymptotic

Manuscript received December 5, 2002; revised January 9, 2003. Recommended by Associate Editor K. Gu.

A. Ilchmann is with the Institute of Mathematics, Technical University Ilmenau, 98693 Ilmenau, Germany (e-mail: ilchmann@mathematik. tu-ilmenau.de).

E. P. Ryan is with the Department of Mathematical Sciences, University of Bath, Bath BA2 7AY, U.K. (e-mail: epr@maths.bath.ac.uk).

Digital Object Identifier 10.1109/TAC.2003.811276 gain values of unnecessarily large magnitude. Clearly, it is of interest to ascertain whether the gain adaptation law can be modified [while still maintaining properties 1) and 2)] in order to restrict the gain growth rate: for example, is it possible to replace the quadratic term in (2) by $\psi(\|y(t)\|)$, where $\psi$ is a bounded function? A corollary (pertaining to linear systems of class $\mathcal{L}$ ) to the main result of the present note answers the latter question affirmatively; for example, the bounded function on the right hand side of the gain adaptation $\dot{k}(t)=\min \left\{\|y(t)\|^{q}, \varepsilon\right\}$ is admissible for every $q>0$ and $\varepsilon>0$.

The overall purpose of this note is to re-examine the above control structure in a more general context of a class $\mathcal{N}_{h}(\mu)$ of nonlinear systems, described by functional differential equations of the form

$$
\left.\begin{array}{rl}
\dot{y}(t) & =f(p(t), y(t),(T y)(t))+g((T y)(t), u(t)) \\
\left.y\right|_{[-h, 0]} & =y^{0} \in C\left([-h, 0] ; \mathbb{R}^{m}\right)
\end{array}\right\}
$$

where, loosely speaking, the parameter $h \geq 0$ quantifies system "memory" and the continuous function $\mu: \mathbb{R}_{\geq 0} \rightarrow \mathbb{R}_{\geq 0}$, with $\mu(0)=0$, relates to the allowable nonlinearities $f(\mu=0$ in the case of systems of the linear class and so $\mathcal{L} \subset \mathcal{N}_{0}(0)$ ). In the context of the class $\mathcal{N}_{h}(\mu)$ (which will be made precise in Section II-A), we establish that the stability properties 1 ) and 2) persist when (2) is replaced by

$$
\left.\begin{array}{l}
u(t)=-k(t)[1+\mu(\|y(t)\|) /\|y(t)\|] y(t) \\
\dot{k}(t)=\psi(\|y(t)\|), \quad k(0)=k^{0} \in \mathbb{R}
\end{array}\right\}
$$

where $\psi: \mathbb{R}_{\geq 0} \rightarrow \mathbb{R}_{\geq 0}$ is any continuous function satisfying

$$
\left.\begin{array}{l}
\text { i) } \psi(s)=0 \text { if, and only if, } s=0 \\
\text { ii) } \underset{s \rightarrow \infty}{\liminf } \frac{s \psi(s)}{s+\mu(s)}>0 \\
\text { iii) } \psi(s)=\mathcal{O}\left(s^{2}+s \mu(s)\right) \text { as } s \downarrow 0
\end{array}\right\} \text {. }
$$

When compared with the strategy for the linear class $\mathcal{L}$, the proportional output feedback law in (2) is augmented by the inclusion of the nonlinear feedback function $y \mapsto \mu(\|y\|)\|y\|^{-1} y$ in (4) to counteract the nonlinearities allowable in (3): by continuity of $\mu$ and since $\mu(0)=0$, this nonlinear feedback function is deemed to take the value zero when $y=0$ and is continuous. The gain adaptation law in (4) may be tailored, through choice of $\psi$, to the needs of a designer to avoid, for example, possible intervals of rapid increase in gain which potentially generate asymptotic gain values of unnecessarily large magnitude (as alluded to earlier in the context of the linear class $\mathcal{L}$ ). Note that ii) is a growth condition at infinity and iii) is a growth condition at zero, each being (loosely speaking) related, via the function $\mu$, to the "strength" of the system nonlinearities. For example, if $\mu(s)=\mathcal{O}(s)$ as $s \rightarrow \infty$, then the bounded function $\psi: s \mapsto \min \left\{s^{2}+s \mu(s), \varepsilon\right\}$ is admissible for every $\varepsilon>0$; or, if $\mu(s)=s^{2}$ (in which case, quadratic nonlinearities are admissible in (3)), then the function $\psi: s \mapsto \min \left\{s^{2}, \varepsilon s\right\}$ is admissible for every $\varepsilon>0$.

\section{ADAPTIVE STABILIZATION}

\section{A. The Class $\mathcal{N}_{h}(\mu)$ of Nonlinear Systems}

Let $h \geq 0$ and let $\mu: \mathbb{R}_{\geq 0} \rightarrow \mathbb{R}_{\geq 0}$ be continuous with $\mu(0)=0$. We now make precise the class $\mathcal{N}_{h}(\mu)$ of nonlinear systems of the form (3) by imposing assumptions on the functions $p, f, g$ and the operator $T$. The class $\mathcal{N}_{h}(\mu)$ is the set of systems of form (3) such that the following holds.

Assumption A: For some $d_{1}, d_{2} \in \mathbb{N}$

1) $p \in L^{2}\left(\mathbb{R}_{\geq 0} ; \mathbb{R}^{d_{1}}\right)$ (with norm denoted by $\|p\|_{L^{2}}$ ); 
2) $f: \mathbb{R}^{d_{1}} \times \mathbb{R}^{m} \times \mathbb{R}^{d_{2}} \rightarrow \mathbb{R}^{m}$ is continuous and there exists $c_{f}>0$ such that

$$
\|f(v, y, w)\| \leq c_{f}[\|v\|+\|y\|+\mu(\|y\|)+\|w\|]
$$

for all $(v, y, w) \in \mathbb{R}^{d_{1}} \times \mathbb{R}^{m} \times \mathbb{R}^{d_{2}}$

3) $g: \mathbb{R}^{d_{2}} \times \mathbb{R}^{m} \rightarrow \mathbb{R}^{m}$ is continuous and there exists a positive-definite, symmetric $G \in \mathbb{R}^{m \times m}$ such that

$$
\langle u, G g(w, u)\rangle \geq\|u\|^{2}
$$

for all $(w, u) \in \mathbb{R}^{d_{2}} \times \mathbb{R}^{m}$

4) $T: C\left([-h, \infty) ; \mathbb{R}^{m}\right) \rightarrow L_{\text {loc }}^{\infty}\left(\mathbb{R}_{\geq 0} ; \mathbb{R}^{d_{2}}\right)$ is a causal operator with the following properties:

i) there exists $c_{T} \geq 0$ such that

$$
\|(T y)(t)\| \leq c_{T} \max _{s \in[-h, t]}\|y(s)\|
$$

for almost all $t \geq 0$ and all $y \in C\left([-h, \infty) ; \mathbb{R}^{m}\right)$;

ii) for each $y \in C\left([-h, \infty) ; \mathbb{R}^{m}\right)$, there exists $c_{y}>0$ such that

$$
\int_{0}^{t}\|(T y)(s)\|^{2} d s \leq c_{y} \int_{-h}^{t}\|y(s)\|^{2} d s \quad \forall t \geq 0
$$

iii) for all $t \geq 0$ and for all continuous $\zeta:[-h, t] \rightarrow$ $\mathbb{R}^{m}$, there exist $\tau, \delta, c>0$ such that, for all $x, \xi \in$ $C\left([-h, \infty) ; \mathbb{R}^{M}\right)$ with $\left.\left.x\right|_{[-h, t]} \equiv \zeta \equiv \xi\right|_{[-h, t]}$ and $x(s)$, $\xi(s) \in \mathbb{B}_{\delta}(\zeta(t))$ for all $s \in[t, t+\tau]$,

$$
\operatorname{esssup}_{s \in[t, t+\tau]}\|(T x)(s)-(T \xi)(s)\| \leq c \sup _{s \in[t, t+\tau]}\|x(s)-\xi(s)\|
$$

where $\mathbb{B}_{\delta}(\zeta(t))$ denotes the open unit ball of radius $\delta>0$ centered at $\zeta(t)$

Remarks 1: We identify (3) with the quadruple $(p, f, g, T)$ and, if Assumption A holds, we write $(p, f, g, T) \in \mathcal{N}(\mu)$.

Assumption A3 is a counterpart of the spectrum condition $\operatorname{spec}(C B) \subset \mathbb{C}_{+}$imposed in the context of the linear class $\mathcal{L}$.

Assumptions A4 i)-ii) essentially form a counterpart of the minimum-phase condition imposed in the context of the linear class. Assumption A4 iii) is a rather weak technical assumption of a local Lipschitz nature imposed to allow application of the existence theory developed in [2].

Example 2 (Finite-Dimensional Linear Prototype): Let $(\tilde{p}, A, B, C)$ define a linear system of class $\mathcal{L}$. Since $C B$ is invertible, $\mathbb{R}^{n}=$ im $B \oplus \operatorname{ker} C$ and there exists $V \in \mathbb{R}^{n \times(n-m)}$, with $\operatorname{im} V=\operatorname{ker} C$, such that

$$
S:=\left[B(C B)^{-1}: V\right]
$$

defines an invertible linear transformation

$$
x \mapsto\left[\begin{array}{l}
y \\
z
\end{array}\right]:=S^{-1} x
$$

which takes (1) into the equivalent form

$$
\left.\begin{array}{l}
\dot{y}(t)=A_{1} y(t)+A_{2} z(t)+C B u(t)+p_{1}(t) \\
\dot{z}(t)=A_{3} y(t)+A_{4} z(t)+p_{2}(t) \\
(y(0), z(0))=\left(y^{0}, z^{0}\right) \\
\operatorname{spec}(C B) \subset \mathbb{C}_{+} \quad \operatorname{spec}\left(-A_{4}\right) \subset \mathbb{C}_{+}
\end{array}\right\}
$$

where the minimum-phase assumption ensures the latter spectrum condition $\operatorname{spec}\left(-A_{4}\right) \subset \mathbb{C}_{+}$(that is, $A_{4}$ is a Hurwitz matrix). Also

$$
\left[\begin{array}{l}
p_{1} \\
p_{2}
\end{array}\right]=S^{-1} \tilde{p} \in L^{2}\left(\mathbb{R}_{\geq 0} ; \mathbb{R}^{n}\right) .
$$

Define the linear operator $T$ and function $p$ by

$$
\left.\begin{array}{rl}
(T y)(t):= & A_{1} y(t) \\
& +A_{2} \int_{0}^{t}\left(\exp A_{4}(t-s)\right) A_{3} y(s) d s \\
p(t):= & p_{1}(t) A_{2}\left(\exp A_{4} t\right) z^{0} \\
& +A_{2} \int_{0}^{t}\left(\exp A_{4}(t-s)\right) p_{2}(s) d s .
\end{array}\right\}
$$

The initial-value problem (6) may now be expressed as

$$
\dot{y}(t)=(T y)(t)+C B u(t)+p(t), \quad y(0)=y^{0}
$$

which is of form (3) with $h=0, f:(v, y, w) \mapsto v+w$ and $g:(w, u) \mapsto C B u$. Since $\tilde{p} \in L^{2}\left(\mathbb{R}_{\geq 0} ; \mathbb{R}^{n}\right)$ and $A_{4}$ is a Hurwitz matrix, it follows that $p$ is in $L^{2}\left(\mathbb{R} \geq_{0} ; \mathbb{R}^{m}\right)$; therefore, Assumption A1 holds. Clearly, Assumption A2 holds with $c_{f}=1$ and $\mu(\cdot) \equiv$ 0 . Since $\operatorname{spec}(C B) \subset \mathbb{C}_{+}$, there exists $G=G^{T}>0$ such that $G C B+(C B)^{T} G=2 I$, whence

$$
\langle u, G g(w, u)\rangle=\langle u, G C B u\rangle=\|u\|^{2} \quad \forall(w, u) \in \mathbb{R}^{d_{2}} \times \mathbb{R}^{m}
$$

and so Assumption A3 holds. Finally, since $A_{4}$ is a Hurwitz matrix, it is readily verified that the operator $T$ satisfies Assumption A4, with $h=0$. Therefore, $(p, f, g, T) \in \mathcal{N}_{0}(0)$ and so the linear class $\mathcal{L}$ is subsumed by $\mathcal{N}_{0}(0)$.

Example 3 (Infinite-Dimensional Regular Linear Systems): The finite-dimensional class of systems of the form (6), considered in Example 2, can be extended to an infinite-dimensional setting by assuming that $p_{1} \in L^{2}\left(\mathbb{R}_{\geq 0} ; \mathbb{R}^{m}\right), p_{2} \in L^{2}\left(\mathbb{R}_{\geq 0} ; X\right)$ ( $X$ a real Hilbert space) and reinterpreting the operators $A_{1}, A_{2}, A_{3}$ and $A_{4}$ as the generating operators of a regular linear system (regular in the sense of [6]). In particular, in this setting, $A_{4}$ is assumed to be the generator of a strongly continuous semigroup $\mathbf{S}=\left(\mathbf{S}_{t}\right)_{t \geq 0}$ of bounded linear operators on the Hilbert space $X$ with norm $\|\cdot\|_{X}$. Let $X_{1}$ denote the space $\operatorname{dom}\left(A_{4}\right)$ endowed with the graph norm and $X_{-1}$ denotes the completion of $X$ with respect to the norm $\|z\|_{-1}=\left\|\left(s_{0} I-A_{4}\right)^{-1} z\right\|_{X}$ where $s_{0}$ is any fixed element of the resolvent set of $A_{4}$. Then, $A_{3}$ is assumed to be a bounded linear operator from $\mathbb{R}^{m}$ to $X_{-1}$ and $A_{2}$ is assumed to be a bounded linear operator from $X_{1}$ to $\mathbb{R}^{m} . A_{1} \in \mathbb{R}^{m \times m}$ is the feedthrough operator of the regular linear system.

If we assume that the semigroup $\mathbf{S}$ is exponentially stable and that the operator $A_{2}$ extends to a bounded linear operator (again denoted by $A_{2}$ ) from $X$ to $\mathbb{R}^{m}$, then the operator $T$ given by

$$
(T y)(t):=A_{1} y(t)+A_{2} \int_{0}^{t} \mathbf{S}_{t-s} A_{3} y(s) d s
$$

satisfies Assumption A4 (for details, see [5]). Moreover, the function

$$
t \mapsto p(t):=p_{1}(t)+A_{2} \mathbf{S}_{t} z^{0}+A_{2} \int_{0}^{t} \mathbf{S}_{t-s} p_{2}(s) d s
$$


is in $L^{2}\left(\mathbb{R}_{\geq 0} ; \mathbb{R}^{m}\right)$ and so Assumption A1 holds. Therefore, system representation (8) carries over to the current infinite-dimensional setting.

Example 4 (Nonlinear Delay Elements): Let functions $\Psi_{n}: \mathbb{R} \times$ $\mathbb{R}^{m} \rightarrow \mathbb{R}^{d_{2}}:(t, y) \mapsto \Psi_{n}(t, y), n=0, \ldots, N$, be measurable in $t$ and globally Lipschitz in $y$ uniformly with respect to $t$ : precisely, i) for each fixed $y, \Psi_{n}(\cdot, y)$ is measurable and ii) there exists a constant $c$ such that

$$
\left\|\Psi_{n}(t, y)-\Psi_{n}(t, z)\right\| \leq c\|y-z\|
$$

for all $t \in \mathbb{R}$ and all $y, z \in \mathbb{R}^{m}$. Assume further that $\Psi_{n}(\cdot, 0) \equiv 0$. For $n=0, \ldots, N$, let $h_{n} \geq 0$ and define $h:=\max _{n} h_{n}$. For $y \in$ $C\left([-h, \infty) ; \mathbb{R}^{m}\right)$, let

$$
(T y)(t):=\int_{-h_{0}}^{0} \Psi_{0}(s, y(t+s)) d s+\sum_{n=1}^{N} \Psi_{n}\left(t, y\left(t-h_{n}\right)\right) \quad \forall t \geq 0 .
$$

The operator $T$, so defined, satisfies Assumption A4; for details, see [5]. Therefore, for example, the system

$$
\begin{aligned}
\dot{y}(t)=L_{1} y(t)+\int_{-h_{0}}^{0} L_{2} y(t+s) d s & \\
& \quad+L_{3} y\left(t-h_{1}\right)+\|y(t)\| L_{4} y(t)+B u(t)
\end{aligned}
$$

with $\operatorname{spec}(B) \subset \mathbb{C}_{+}$and matrices $L_{i} \in \mathbb{R}^{m \times m}(i=1, \ldots, 4)$, is of class $\mathcal{N}_{h}(\mu)$, where $h:=\max \left\{h_{0}, h_{1}\right\}$ and $\mu: s \mapsto s^{2}$.

\section{B. Stability Analysis}

We now arrive at the main result.

Theorem 5: Let $h \geq 0$, let $\mu: \mathbb{R}>0 \rightarrow \mathbb{R}_{>0}$ be continuous with $\mu(0)=0$, and let $\psi: \mathbb{R}_{>0} \rightarrow \mathbb{R}_{>0}$ be continuous and such that (5) holds. Let $(p, f, g, T) \in \mathcal{N}_{h}(\mu)$ and $\left(y^{0}, k^{0}\right) \in C\left([-h, 0] ; \mathbb{R}^{m}\right) \times \mathbb{R}$. Then, the application of (4) to (3), with initial data $y^{0} \in C\left([-h, 0] ; \mathbb{R}^{m}\right)$, yields the closed-loop initial-value problem

$$
\begin{aligned}
\dot{y}(t)= & f(p(t), y(t),(T y)(t)) \\
& +g((T y)(t),-k(t) \\
& \left.\times\left[1+\mu(\|y(t)\|)\|y(t)\|^{-1}\right] y(t)\right) \\
\dot{k}(t)= & \psi(\|y(t)\|) \\
\left.(y, k)\right|_{[-h, 0]}= & \left(y^{0}, k^{0}\right)
\end{aligned}
$$

with the following properties.

I) There exists a solution of (10) and every solution can be extended to a maximal solution.

II) Every maximal solution $(y, k):[-h, \omega) \rightarrow \mathbb{R}^{m} \times \mathbb{R}$ of (10) is such that

i) $\omega=\infty$;

ii) $\lim _{t \rightarrow \infty} k(t)$ exists and is finite;

iii) $y(t) \rightarrow 0$ as $t \rightarrow \infty$.

Proof: That (10) has a solution and every solution has a maximal extension follow from [2, Th. 2.3] (see also the first paragraph of the proof of [2, Th. 3.2]).

Let $(y, k):[-h, \omega) \rightarrow \mathbb{R}^{m} \times \mathbb{R}$ be a maximal solution of (10). Let $G=G^{T}>0$ be such that the inequality in Assumption A3 holds. Define the norm $\|\cdot\|_{G}$ on $\mathbb{R}^{m}$ by $\|u\|_{G}:=\langle u, G u\rangle$ and note the inequalities

$$
\left\|G^{-1}\right\|^{-1}\|u\|^{2} \leq\|u\|_{G}^{2} \leq\|G\|\|u\|^{2} \quad \forall u \in \mathbb{R}^{m} .
$$

By Assumptions A2 and A3

$$
\begin{aligned}
& \frac{d}{d t}\|y(t)\|_{G}^{2} \\
& =2\langle y(t), G[f(p(t), y(t),(T y)(t)) \\
& \left.\left.\quad+g\left((T y)(t),-k(t)\left[1+\mu(\|y(t)\|)\|y(t)\|^{-1}\right] y(t)\right)\right]\right\rangle \\
& \leq 2 c_{f}\|G\|\|y(t)\|[\|p(t)\|+\|y(t)\|+\mu(\|y(t)\|) \\
& \quad+\|(T y)(t)\|]-2 k(t)[\|y(t \|+\mu(\|y(t)\|)]\| y(t) \| \\
& \leq c_{f}\|G\|\left[\|p(t)\|^{2}+4\|y(t)\|^{2}+2 \mu(\|y(t)\|)\|y(t)\|\right. \\
& \left.\quad+\|(T t)(t)\|^{2}\right]-2 k(t)\left[\|y(t)\|^{2}+\mu(\|y(t)\|)\|y(t)\|\right] \\
& \quad \text { for almost all (a.a.) } t \in[0, \omega)
\end{aligned}
$$

and so, invoking (11), there exists a constant $c_{1}>0$ such that

$$
\begin{aligned}
\frac{d}{d t} \| y & (t) \|_{G}^{2} \leq-\left[c_{1}^{-1} k(t)-c_{1}\right] \\
& \times\left[\|y(t)\|_{G}^{2}+\mu(\|y(t)\|)\|y(t)\|\right] \\
& +c_{1}\left[\|p(t)\|^{2}+\|(T y)(t)\|^{2}\right] \text { a.a. } t \in[0, \omega) .
\end{aligned}
$$

By integration, together with (11) and Assumptions A1 and A4 ii), we may conclude the existence of a constant $c_{2}>c_{1}\left[\|p\|_{L^{2}}^{2}+1\right]$ such that

$$
\begin{aligned}
&\|y(t)\|_{G}^{2} \leq\|y(\tau)\|_{G}^{2}+c_{2}+c_{2} \int_{-h}^{\tau}\|y(s)\|_{G}^{2} d s \\
&-\int_{\tau}^{t} \alpha(s)\left[\|y(s)\|_{G}^{2}+\mu(\|y(t)\|)\|y(s)\|\right] d s \\
& \forall t, \tau, 0 \leq \tau \leq t<\omega
\end{aligned}
$$

wherein, for notational convenience, we have introduced the nondecreasing function $\alpha$ given by

$$
\alpha(s):=c_{1}^{-1} k(s)-c_{2} .
$$

The proof of Assertion II) now proceeds in three steps. First, by a contradiction argument, we show that $k$ is bounded. Second, we prove that $y$ is also bounded and so $\omega=\infty$, whence Assertion II-i) and, by boundedness and monotonicity of $k$, Assertion II-ii). Finally, we establish Assertion II-iii).

Step 1) For contradiction, suppose that $k$ is unbounded. Choose $\tau \in$ $[0, \omega)$ such that

$$
\alpha(\tau)=c_{1}^{-1} k(\tau)-c_{2} \geq 1
$$

Then, by (15)

$\|y(t)\|_{G}^{2} \leq\|y(\tau)\|_{G}^{2}+c_{2}+c_{2} \int_{-h}^{\tau}\|y(s)\|_{G}^{2} d s=: \beta \quad \forall t \in[\tau, \omega)$.

By continuity of $y$, we conclude that $y \in L^{\infty}\left([-h, \omega) ; \mathbb{R}^{m}\right)$ and so, by continuity of $\psi$, we may infer boundedness of $\dot{k}(\cdot)=\psi(\|y(\cdot)\|)$. By the supposition of unboundedness of $k$, it follows that $\omega=\infty$.

By (14) and (17), together with monotonicity of $\alpha$, boundedness of $y$ and Assumption A4-i), we may conclude the existence of $c_{3} \geq c_{1}$ such that

$$
\frac{d}{d t}\|y(t)\|_{G}^{2} \leq-\alpha\left(t_{0}\right)\|y(t)\|_{G}^{2}+c_{3}\left[\|p(t)\|^{2}+1\right]
$$


for almost all $t_{0}, t$ with $\tau \leq t_{0} \leq t$. Integration yields

$$
\begin{aligned}
\|y(t)\|_{G}^{2} \leq & e^{-\alpha\left(t_{0}\right)\left(t-t_{0}\right)}\left\|y\left(t_{0}\right)\right\|_{G}^{2} \\
& +\int_{t_{0}}^{t} e^{-\alpha\left(t_{0}\right)(t-s)} c_{3}\left[\|p(s)\|^{2}+1\right] d s \\
\leq & e^{-\alpha\left(t_{0}\right)\left(t-t_{0}\right)} \sup _{t \geq-h}\|y(t)\|_{G}+\frac{c_{3}}{\alpha\left(t_{0}\right)} \\
& +c_{3} \int_{t_{0}}^{t} e^{-\alpha\left(t_{0}\right)(t-s)}\|p(s)\|^{2} d s
\end{aligned}
$$

for all $t, t_{0}$ with $\tau \leq t_{0} \leq t$. Now, as a convolution of the $L^{1}$ functions $t \mapsto e^{-\alpha\left(t_{0}\right) t}$ and $t \mapsto\|p(t)\|^{2}$, we have

$$
\begin{aligned}
& \int_{t_{0}}^{t} e^{-\alpha\left(t_{0}\right)(t-s)}\|p(s)\|^{2} d s \\
& \leq\left(\int_{t_{0}}^{\infty} e^{-\alpha\left(t_{0}\right) t} d t\right)\left(\int_{t_{0}}^{\infty}\|p(t)\|^{2} d t\right)<\frac{\|p\|_{L^{2}}^{2}}{\alpha\left(t_{0}\right)}
\end{aligned}
$$

for all $t \geq t_{0} \geq \tau$. Therefore

$$
\left\|y\left(t_{0}+1\right)\right\|_{G}^{2} \leq e^{-\alpha\left(t_{0}\right)} \sup _{t \geq-h}\|y(t)\|_{G}+\frac{c_{3}\left[\|p\|_{L_{2}}^{2}+1\right]}{\alpha\left(t_{0}\right)}
$$

for all $t_{0} \geq \tau$. Since $\alpha\left(t_{0}\right) \rightarrow \infty$ as $t_{0} \rightarrow \infty$, it follows that $\lim _{t \rightarrow \infty} y(t)=0$.

Invoking (11), (17), monotonicity of $\alpha$ and (15), together with the definition of $\beta$ in (18), we have

$$
\begin{aligned}
& \int_{\tau}^{t}\left[\|y(s)\|^{2}+\mu(\|y(s)\|)\|y(s)\|\right] d s \\
& \quad \leq\left(1+\left\|G^{-1}\right\|\right) \int_{\tau}^{t}\left[\|y(s)\|_{G}^{2}+\mu(\|y(s)\|)\|y(s)\|\right] d s \\
& \quad \leq\left(1+\left\|G^{-1}\right\|\right) \int_{\tau}^{t} \alpha(s)\left[\|y(s)\|_{G}^{2}+\mu(\|y(s)\|)\|y(s)\|\right] d s \\
& \quad \leq\left(1+\left\|G^{-1}\right\|\right) \beta \quad \forall t \in[\tau, \infty) .
\end{aligned}
$$

Therefore

$$
\|y(\cdot)\|^{2}+\mu(\|y(\cdot)\|)\|y(\cdot)\| \in L^{1}\left([-h, \infty) ; \mathbb{R}^{m}\right) .
$$

Recalling that $y(t) \rightarrow 0$ as $t \rightarrow \infty$ and invoking property (5) iii) of $\psi$, there exists $t^{*}>0$ and $K>0$ such that

$$
\psi(\|y(t)\|) \leq K\left[\|y(t)\|^{2}+\mu(\|y(t)\|)\|y(t)\|\right] \forall t \geq t^{*} .
$$

Hence, $\dot{k}(\cdot)=\psi(\|y(\cdot)\|) \in L^{1}([-h, \infty) ; \mathbb{R})$ which contradicts the supposition of unboundedness of $k$. Therefore, $k$ is bounded.

Step 2) For contradiction, suppose that the function $y:[-h, \omega) \rightarrow$ $\mathbb{R}^{m}$ is unbounded. For each $n \in \mathbb{N}$, define

$$
\begin{aligned}
& \tau_{n}:=\inf \left\{t \in[0, w) \mid\|y(t)\|_{G}^{2}=n+1+\|y(0)\|_{G}^{2}\right\} \\
& \sigma_{n}:=\sup \left\{t \in\left[0, \tau_{n}\right) \mid\|y(t)\|_{G}^{2}=n+\|y(0)\|_{G}^{2}\right\} .
\end{aligned}
$$

Note that

$$
\begin{aligned}
& \max _{s \in[0, t]}\|y(s)\|^{2} \leq\|G\|^{-1} \max _{s \in[0, t]}\|y(s)\|_{G}^{2} \\
& \quad \leq\|G\|^{-1}\left(n+1+\|y(0)\|_{G}^{2}\right) \\
& \quad \leq 2\|G\|^{-1}\left(n+\|y(0)\|_{G}^{2}\right) \\
& \quad \leq 2\|G\|^{-1}\|y(t)\|_{G}^{2} \forall t \in\left[\sigma_{n}, \tau_{n}\right] \forall n \in \mathbb{N}
\end{aligned}
$$

and so, invoking Assumption A4-i), there exists a constant $c_{4}>0$ such that

$$
\|(T y)(t)\|^{2} \leq c_{4}\|y(t)\|_{G}^{2} \quad \forall t \in\left[\sigma_{n}, \tau_{n}\right] \quad \forall n \in \mathbb{N} .
$$

In passing, we also note that

$$
1 \leq\|y(t)\|_{G} \quad \forall t \in\left[\sigma_{n}, \tau_{n}\right] \quad \forall n \in \mathbb{N} .
$$

We may now infer, from (14) together with boundedness of $k$, the existence of a constant $c_{5}>0$ such that

$$
\begin{aligned}
\frac{d}{d t}\|y(t)\|_{G}^{2} \leq & c_{5}\left[\|y(t)\|_{G}^{2}+\mu(\|y(t)\|)\|y(t)\|\right]+c_{1}\|p(t)\|^{2} \\
\leq & \left(c_{5}\left[1+\mu(\|y(t)\|)\|y(t)\|\|y(t)\|_{G}^{-2}\right]\right. \\
& \left.+c_{1}\|p(t)\|^{2}\right)\|y(t)\|_{G}^{2} \\
& \forall t \in\left[\sigma_{n}, \tau_{n}\right] \forall n \in \mathbb{N} .
\end{aligned}
$$

By property (5) ii) of the continuous function $\psi$, there exists $\varepsilon>0$ so that

$$
\psi(s) \geq \varepsilon\left[1+\frac{\mu(s)}{s}\right], \quad \text { for all } s>0 \text { sufficiently large }
$$

which, in conjunction with (11) and the fact that $\|y(t)\|_{G}^{2} \geq n+$ $\|y(0)\|_{G}^{2}$ for all $t \in\left[\sigma_{n}, \tau_{n}\right]$ and all $n \in \mathbb{N}$, implies the existence of $N \in \mathbb{N}$ such that

$$
\begin{aligned}
1+ & \mu(\|y(t)\|)\|y(t)\|\|y(t)\|_{G}^{-2} \\
& \leq 1+\left\|G^{-1}\right\| \mu(\|y(t)\|)\|y(t)\|^{-1} \\
& \leq\left[1+\left\|G^{-1}\right\|\right]\left[1+\mu(\|y(t)\|)\|y(t)\|^{-1}\right] \\
& \leq \epsilon^{-1}\left[1+\left\|G^{-1}\right\|\right] \psi(\|y(t)\|) \forall t \in\left[\sigma_{n}, \tau_{n}\right] \quad \forall n \geq N .
\end{aligned}
$$

Writing $c_{6}:=c_{5} \varepsilon^{-1}\left[1+\left\|G^{-1}\right\|\right]>0$, then, by (20), it follows that $\frac{d}{d t} \ln \|y(t)\|_{G}^{2}=\|y(t)\|_{G}^{-2} \frac{d}{d t}\|y(t)\|_{G}^{2}$

$$
\leq c_{6} \psi(\|y(t)\|)+c_{1}\|p(t)\|^{2} \forall t \in\left[\sigma_{n}, \tau_{n}\right] \forall n \geq N .
$$

Therefore, by integration

$$
\begin{aligned}
\ln \left\|y\left(\tau_{n}\right)\right\|_{G}^{2} & -\ln \left\|y\left(\sigma_{n}\right)\right\|_{G}^{2} \\
& \leq c_{6} \int_{\sigma_{n}}^{\tau_{n}} \psi(\|y(t)\|) d t+c_{1} \int_{\sigma_{n}}^{\tau_{n}}\|p(t)\|^{2} d t \quad \forall n \geq N
\end{aligned}
$$

which, in turn, implies that

$$
\begin{aligned}
& \ln \left(\frac{M+1+\|y(0)\|_{G}^{2}}{N+\|y(0)\|_{G}^{2}}\right)=\ln \left\|y\left(\tau_{M}\right)\right\|_{G}^{2}-\ln \left\|y\left(\sigma_{N}\right)\right\|_{G}^{2} \\
& \quad=\sum_{n=N}^{M}\left[\ln \left\|y\left(\tau_{n}\right)\right\|_{G}^{2}-\ln \left\|y\left(\sigma_{n}\right)\right\|_{G}^{2}\right] \\
& \quad \leq c_{6} \sum_{n=N}^{M} \int_{\sigma_{n}}^{\tau_{n}} \psi(\|y(t)\|) d t+c_{1} \sum_{n=N_{\sigma_{n}}}^{M} \int_{\sigma_{n}}^{\tau_{n}}\|p(s)\|^{2} d s \\
& \quad=c_{6}\left[k\left(\tau_{M}\right)-k\left(\sigma_{N}\right)\right]+c_{1}\|p\|_{L^{2}}^{2} \quad \forall M \geq N .
\end{aligned}
$$

Since $k$ is bounded, the right-hand side of (21) is bounded, contradicting the fact that the left-hand side tends to infinity as $M \rightarrow \infty$. Therefore, the supposition of unboundedness of $y$ is false and so $y \in$ $L^{\infty}\left([-h, \omega) ; \mathbb{R}^{m}\right)$.

By boundedness of $(y, k)$ on $[0, \omega)$ and maximality of $\omega$ it follows that $\omega=\infty$, where Assertion II-i) and, by boundedness and monotonicity of $k$, Assertion II-ii) immediately follows.

Step 3: Again seeking a contradiction, suppose that $y(t) \nrightarrow 0$ as $t \rightarrow \infty$. Then, there exists $\delta>0$ and an unbounded $\mathbb{R}_{\geq 0}$-valued sequence $\left(s_{n}\right)$ such that $\left\|y\left(s_{n}\right)\right\| \geq 3 \delta$ for all $n \in \mathbb{N}$. Define $\Delta:=\inf \{\psi(s) \mid s>\delta\}$. By properties of $\psi$, we have $\Delta>0$. Since $\psi(\|y(\cdot)\|)$ is of class $L^{1}$, there exists an unbounded $\mathbb{R}_{\geq 0}$-valued 
sequence $\left(t_{n}\right)$ such that $\psi\left(\left\|y\left(t_{n}\right)\right\|\right)<\Delta$ for all $n \in \mathbb{N}$ and so, by definition of $\Delta$, we have $\left\|y\left(t_{n}\right)\right\| \leq \delta$ for all $n \in \mathbb{N}$. Extracting subsequences if necessary, we may assume $t_{n} \in\left(s_{n}, s_{n+1}\right)$ for all $n \in \mathbb{N}$. By continuity of $y$ and since $\left\|y\left(s_{n}\right)\right\| \geq 3 \delta$ and $\left\|y\left(t_{n}\right)\right\| \leq \delta$, for each $n \in \mathbb{N}$, there exists $r_{n} \in\left(s_{n}, t_{n}\right)$ such that $\left\|y\left(r_{n}\right)\right\|=2 \delta$. Again extracting a subsequence if necessary, we may assume $r_{n+1}-r_{n} \geq 1$ for all $n \in \mathbb{N}$. By the first of equations (10) and Assumption A, together with boundedness of the solution $(y, k)$, there exists a constant $c_{7}>0$ such that

$$
\|\dot{y}(t)\| \leq c_{7}(1+\|p(t)\|) \quad \text { for a.a. } t \geq 0
$$

An application of Hölder's inequality yields

$$
\begin{array}{r}
\int_{t}^{t+\tau}(1+\|p(s)\|) d s=\tau+\int_{t}^{t+\tau}\|p(s)\| d s \leq \tau+\sqrt{\tau}\|p\|_{L^{2}} \\
\forall t \geq 0 \forall \tau>0 .
\end{array}
$$

Choose $\tau \in(0,1)$ sufficiently small so that

$$
\tau+\sqrt{\tau}\|p\|_{L^{2}}<\frac{\delta}{c_{7}}
$$

in which case, we have

$$
\|y(t+\sigma)-y(t)\| \leq \int_{t}^{t+\tau}\|\dot{y}(s)\| d s<\delta
$$

for all $t \in \mathbb{R}_{\geq 0}$ and all $\sigma \in[0, \tau]$. Therefore

$$
\begin{aligned}
\left\|y\left(r_{n}+\sigma\right)\right\| \geq\left\|y\left(r_{n}\right)\right\|-\left\|y\left(r_{n}+\sigma\right)-y\left(r_{n}\right)\right\| \\
=2 \delta-\left\|y\left(r_{n}+\sigma\right)-y\left(r_{n}\right)\right\|>\delta
\end{aligned}
$$

for all $\sigma \in\left[r_{n}, r_{n}+\tau\right]$ and all $n \in \mathbb{N}$, and so

$$
\psi(\|y(t)\|) \geq \Delta \forall t \in \cup_{n \in N}\left[r_{n}, \tau_{n}+\tau\right]
$$

which (on noting that the intervals $\left[r_{n}, r_{n}+\tau\right], n \in \mathbb{N}$, are each of length $\tau>0$ and form a mutually disjoint family) contradicts the fact that $\psi(\|y(\cdot)\|)$ is of class $L^{1}$. Therefore, $y(t) \rightarrow 0$ as $t \rightarrow \infty$. This completes the proof.

Example 6: Let $h \geq 0$ and $\mu: s \mapsto s^{q}$, with $q>0$. Define

$$
q_{0}:=\min \{2, q+1\} \quad q_{\infty}:=\max \{1, q\}-1
$$

Then, for each $\varepsilon>0$

$$
\begin{aligned}
& u(t)=-k(t)\left[1+\|y(t)\|^{q-1}\right] y(t) \\
& \dot{k}(t)=\min \left\{\|y(t)\|^{q_{0}}, \epsilon\|y(t)\|^{q_{\infty}}\right\},\left.\quad k\right|_{[-h, 0]}=k^{0}
\end{aligned}
$$

defines an $\mathcal{N}_{h}(\mu)$-universal strategy.

\section{Linear Subclass $\mathcal{L} \subset \mathcal{N}_{0}(0)$ Revisited}

Inspection of the proof of Theorem 5 reveals that property iii) in (5) plays a rôle only in Step 1: in particular, it is shown therein that, if $k$ is unbounded (in which case $\omega=\infty$ ), then, by property iii) in (5), $\psi(\|y(\cdot)\|) \in L^{1}\left(\mathbb{R}_{\geq 0} ; \mathbb{R}^{m}\right)$. Now, a well-known high-gain property of linear systems of class $\mathcal{L}$ implies that, if $k$ is unbounded, then $y$ decays exponentially to zero and so the requisite conclusion $\psi(\|y(\cdot)\|) \in$ $L^{1}\left(\mathbb{R}_{\geq 0} ; \mathbb{R}^{m}\right)$ still holds if property iii) in (5) (with $\mu=0$ in the current context of the system class $\left.\mathcal{L} \subset \mathcal{N}_{0}(0)\right)$ is replaced by the following weaker property: for some $q>0, \psi(s)=\mathcal{O}\left(s^{q}\right)$ as $s \downarrow 0$.

Therefore, we may conclude the following.

Corollary 7: Let $\psi: \mathbb{R}_{\geq 0} \rightarrow \mathbb{R}_{\geq 0}$ be continuous and such that

$$
\left.\begin{array}{l}
\text { i) } \psi(s)=0 \text { if, and only if, } s=0 \\
\text { ii) } \liminf _{s \rightarrow \infty} \psi(s)>0 \\
\text { iii') for some } q>0, \psi(s)=\mathcal{O}\left(s^{q}\right) \text { as } s \downarrow 0
\end{array}\right\} \text {. }
$$

Let $(p, A, B, C) \in \mathcal{L}$ and $\left(x^{0}, k^{0}\right) \in \mathbb{R}^{n} \times \mathbb{R}$. Then, application of the control

$$
u(t)=-k(t) y(t) \quad \dot{k}=\psi(\|y(t)\|), \quad k(0)=k^{0} \in \mathbb{R}
$$

to system (1) yields a closed-loop initial-value problem with the following properties.

I) There exists a solution and every solution can be maximally extended.

II) Every maximal solution $(x, k):[0, \omega) \rightarrow \mathbb{R}^{n} \times \mathbb{R}$ is such that

$$
\begin{aligned}
& \text { i) } \omega=\infty \text {; } \\
& \text { ii) } \lim _{t \rightarrow \infty} k(t) \text { exists and is finite; } \\
& \text { iii) } x(t) \rightarrow 0 \text { as } t \rightarrow \infty \text {. }
\end{aligned}
$$

Proof: Invoking Example 2, modifying Step 1) of the proof of Theorem 5 as indicated above, and applying Step 2), Assertions I and II i-ii) readily follow. Moreover, the argument in Step 3) also applies to conclude $y(t) \rightarrow 0$ as $t \rightarrow \infty$. Consider the equivalent representation of (1) given by (6). Since $p_{2} \in L^{2}\left(\mathbb{R}_{\geq 0} ; \mathbb{R}^{m}\right)$, $\operatorname{spec}\left(-A_{4}\right) \subset \mathbb{C}_{+}$and $y(t) \rightarrow 0$ as $t \rightarrow \infty$, it follows that $z(t) \rightarrow 0$ as $t \rightarrow \infty$ and so

$$
x(t)=S\left[\begin{array}{l}
y(t) \\
z(t)
\end{array}\right] \rightarrow 0 \text { as } t \rightarrow \infty .
$$

This completes the proof.

Example 8: For every $q>0$ and $\varepsilon>0$,

$$
u(t)=-k(t) y(t) \quad \dot{k}(t)=\min \left\{\|y(t)\|^{q}, \varepsilon\right\}, \quad k(0)=k^{0}
$$

is an $\mathcal{L}$-universal feedback strategy.

\section{REFERENCES}

[1] C. I. Byrnes and J. C. Willems, "Adaptive stabilization of multivariable linear systems," in Proc. 23rd Conf. Decision Control, Las Vegas, NV, 1984, pp. 1574-1577.

[2] A. Ilchmann, E. P. Ryan, and C. J. Sangwin, "Systems of controlled functional differential equations and adaptive tracking," SIAM J. Control Optim., vol. 40, pp. 1746-1764, 2002.

[3] I. Mareels, "A simple selftuning controller for stably invertible systems," Syst. Control Lett., vol. 4, pp. 5-16, 1984.

[4] A. S. Morse, "Recent problems in parameter adaptive control," in Outils et Modèles Mathématiques pour l'Automatique, l'Analyze de Systèmes et le Traitment du Signal, I. D. Landau, Ed., Paris, France, 1983, pp. 733-740.

[5] E. P. Ryan and C. J. Sangwin, "Controlled functional differential equations and adaptive stabilization," Int. J. Control, vol. 74, pp. 77-90, 2001.

[6] G. Weiss, "Transfer functions of regular linear systems, part 1: characterization of regularity," Trans. Amer. Math. Soc., vol. 342, pp. 827-854, 1994. 